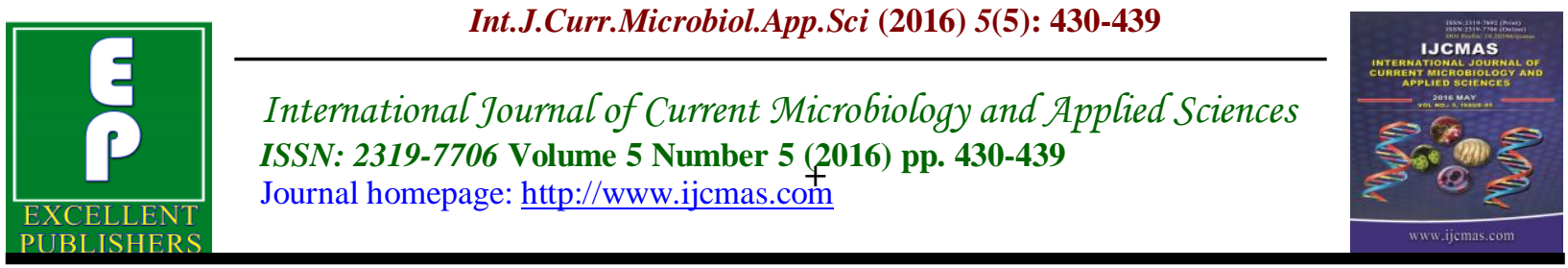

Original Research Article

http://dx.doi.org/10.20546/ijcmas.2016.505.045

\title{
Prevalence and Antimicrobial Susceptibility of Extended Spectrum $\beta$-Lactamases (ESBL) Producing Escherichia coli and Klebsiella pneumoniae Isolates in a Tertiary Care Hospital in North-West India
}

\author{
Saroj Hooja*, Nita Pal, Rajendra Karadiya, Rajni Sharma, \\ Ramesh Mishra and Rakesh Maheshwari \\ Department of Microbiology SMS Medical College, Jaipur, India \\ *Corresponding author
}

\begin{tabular}{l} 
K e y w o r d s \\
DDST, \\
Escherichia coli, \\
extended spectrum \\
$\beta$-lactamases, \\
Klebsiella \\
pneumoniae, \\
PCDDT. \\
\hline Article Info \\
\hline Accepted: \\
15 April 2016 \\
Available Online: \\
10 May 2016 \\
\end{tabular}

A B S T R A C T

\begin{abstract}
Infections caused by extended spectrum $\beta$-lactamases (ESBL) producing gram negative bacteria complicate therapy and limit treatment options. The aim of the present study was to determine the prevalence and susceptibility of ESBL Escherichia coli (E. coli) and Klebsiella pneumoniae from various clinical isolates among hospitalized and outdoor patients at our tertiary care hospital. A total of 412 isolates from various clinical samples were screened for ESBL as per CLSI guidelines and confirmed by Phenotypic Confirmatory Disc Diffusion Test (PCDDT) and Double Disc Synergy Test (DDST). Antimicrobial susceptibility of these isolates was performed by Kirby- Bauer disc diffusion method. ESBL production was seen in 174/281(61.92\%) of E. coli and 74/131(56.48\%) of Klebsiella pneumoniae isolates. PCDDT detected 174(61.3\%) and 74(56.5\%) of ESBL while DDST detected $164(58.4 \%)$ and 70 (53.4\%) of ESBL E. coli and Klebsiella pneumoniae respectively. ESBL producers were higher among inpatients 199/323(61.60\%) compared to outpatients 49/89(55.05\%). ESBL production was found to be highest among blood isolates. A high level of resistance was seen with third generation cephalosporins, piperacillin and cefepime in both ESBL E. coli and Klebsiella pneumoniae isolates. Strict adherence to the hospital antibiotic policy and good infection control practices would go a long way in curtailing the menace of drug resistance.
\end{abstract}

\section{Introduction}

Extended-spectrum $\beta$-lactamases (ESBLs) are a rapidly evolving group of $\beta$-lactamases which share the ability to hydrolyze thirdgeneration cephalosporins and aztreonam yet are inhibited by clavulanic acid. (Paterson, 2005) The majority of ESBLs are derived from the widespread broadspectrum $\beta$-lactamases SHV-1 and TEM-1.
There are also new families of ESBLs, including the CTX-M and OXA-type enzymes as well as novel, unrelated $\beta$ lactamases. (Bradford, 2001) Typically, they are derived by mutations of these genes that alter the amino acid configuration around the active site of these $\beta$-lactamases. The presence of ESBLs carries tremendous clinical significance. ESBLs are often 
encoded by genes located on large plasmids that also carry genes for resistance to other antimicrobial agents such as aminoglycosides, trimethoprim, sulphonamides, tetracyclines and chloramphenicol. (Paterson, 2000) They are most commonly produced by Klebseilla spp. and Escherichia coli. However, Enterobacter, Salmonella, Proteus, Citrobacter, Morganella, Serratia, Shigella and Pseudomonas also produce them (Paterson, 2006).

They are distributed worldwide and within geographic areas and are rapidly changing over time.( Kenneth, 2008) Infections with ESBL producing organisms continue to be associated with limited therapeutic options resulting in higher mortality and morbidity rate as well as high cost of treatment.

The aim of the present study was to determine the frequency and antimicrobial susceptibility pattern among various clinical isolates of ESBL producing $E$. coli and Klebsiella pneumoniae from samples collected from hospitalized patients and those attending outpatient clinics at our tertiary care hospital.

\section{Materials and Methods}

This prospective study was performed over a period of one year from June 2013 to May 2014 at SMS Medical College, Jaipur. A total of 412 consecutive non-duplicate $E$. coli and Klebsiella pneumoniae isolates recovered from various clinical samples from patients either admitted to different wards or attending the outpatient departments were included.

Patient's demographic data, clinical diagnosis and specimen types were recorded. Only one positive culture per patient was included in the study. The isolates were identified to species level using standard microbiological techniques.

\section{Antimicrobial Susceptibility Testing}

All isolates were examined for susceptibility to cefepime $(30 \mu \mathrm{g})$, ceftriaxone $(30 \mu \mathrm{g})$, ceftazidime $(30 \mu \mathrm{g})$, cefotaxime $(30 \mu \mathrm{g})$, piperacillin $(30 \mu \mathrm{g})$, amikacin $(30 \mu \mathrm{g})$, gentamicin $(10 \mu \mathrm{g})$, cefuroxime $(30 \mu \mathrm{g})$, ciprofloxacin $(5 \mu \mathrm{g})$, doxycycline $(30 \mu \mathrm{g})$, meropenem $(10 \mu \mathrm{g})$, norfloxacin $(10 \mu \mathrm{g})$, nitrofurantoin $(300 \mu \mathrm{g})$ and cefoperazone/ sulbactam $(75 / 10 \mu \mathrm{g})$ using commercially available discs (Hi media, Mumbai, India) by disc diffusion method as per Clinical and Laboratory Standards Institute (CLSI) guidelines. (Clinical Laboratory Standards Institute, 2015.) Isolates showing reduced zone of inhibition to third generation cephalosporins ie. ceftazidime $30 \mu \mathrm{g}$ (zone $\leq$ $22 \mathrm{~mm}$ ) and cefotaxime (zone $\leq 27 \mathrm{~mm}$ ) as recommended by CLSI guidelines were considered as potential ESBL producers and were selected for confirmation for ESBL production.

\section{ESBL Detection: Phenotypic Confirmatory Disc Diffusion Test (PCDDT)}

Isolates were characterized phenotypically for ESBL production using phenotypic confirmatory test as recommended by CLSI. (Clinical Laboratory Standards Institute, 2015.) The test was done using both cefotaxime $(30 \mu \mathrm{g})$ and ceftazidime $(30 \mu \mathrm{g})$ alone and in combination with clavulanic acid. A $>5 \mathrm{~mm}$ increase in the inhibition zone diameter for either antimicrobial agent tested in combination with clavulanic acid versus its zone when tested alone was taken as positive result for ESBL production. (Fig1)

\section{Double Disc Synergy Test (DDST)}

A 0.5 McFarland suspension of the test isolate was swabbed on Muller Hinton Agar (MHA) plate and $30 \mu \mathrm{g}$ antibiotic discs of 
ceftazidime, ceftriaxone and cefotaxime were placed on the plate $15 \mathrm{~mm}$ (center to center) from the amoxycillin/clavulanate $(20 \mu \mathrm{g} / 10 \mu \mathrm{g})$ (augmentin) disc and incubated at 37 0C aerobically overnight. (Jarlier et al., 1988) Enhacement of the inhibition zone of any of these cephalosporin discs towards the augmentin disc was interpreted as positive for ESBL production. (Fig2)

Quality control: Klebsiella pneumoniae ATCC 700603 (positive control) and E. coli ATCC 25922 (negative control) were used for quality control of ESBL tests.

Statistical Analysis: Chi square test was applied for analysis of categorical data. All statistical calculations were done by using MedCalc Statistical Software version 14.12.0(Medcalc Software bvba,Medcalc Ostend, Belgium). $\mathrm{P}<0.05$ was taken as significant for interpretation.

\section{Results and Discussion}

Out of 412 isolates that were included in the study, 281 were E. coli and 131 were Klebsiella pneumoniae. The overall prevalence of ESBL producing isolates was 248/412(60.2\%) as detected by PCDDT while DDST detected only 234 ESBL producers. ESBL production was seen in $174 / 281(61.9 \%)$ of E. coli and $74 / 131(56.5 \%)$ of Klebsiella pneumoniae. ESBL producers were higher among isolates from inpatients 199/323 (61.6\%) compared to those from outpatients 49/89(55.0\%). Maximum ESBL producers were isolated from intensive care unit (ICU) patients $35 / 50(70.0 \%)$, followed by pediatric ward $36 / 54(66.7 \%)$ and surgical ward 84/132(63.6\%). (Table1) The age and sexwise distribution revealed that maximum numbers of ESBL producers were seen in the age group 0-14 years. (Table2) ESBL producing isolates were more among females than males. ESBL E. coli was most commonly isolated from patients of bacteremia10/12(83.3\%), followed by respiratory tract infection $8 / 11(72.7 \%$ ) and urinary tract infection142/220(64.5\%). ESBL Klebsiella pneumoniae was most commonly isolated from patients of bacteremia 42/53(78.0\%) followed by urinary tract infection $10 / 17(58.8 \%)$ and skin and soft tissue infections 18/33(54.5\%). (Table3)

\section{Antimicrobial Resistance Pattern}

A wide spectrum of antimicrobial resistance pattern to various antimicrobial agents was observed in ESBL E. coli and Klebsiella pneumoniae. Among the ESBL E. coli a high $(>80 \%)$ degree of resistance was observed with 3rd generation cephalosporins (3GCs), cefepime, cefuroxime and piperacillin. Resistance to other classes of antimicrobials was also observed. A resistance of $51.1 \%$ each to doxycycline and ciprofloxacin, $40.8 \%$ to gentamicin, $33.90 \%$ to cefaperazone/sulbactam and $16.7 \%$ to amikacin was observed. Meropenem showed the least resistance of $1.1 \%$. (Fig3) Among the urinary isolates a resistance of $17.0 \%$ and $89.4 \%$ was observed with nitrofurantoin and norfloxacin respectively. Among the ESBL Klebsiella pneumoniae a high (>85\%) degree of resistance was observed with 3GCs, cefepime, cefuroxime and piperacillin. Among the aminoglycosides a resistance of $52.7 \%$ was observed each with amikacin and gentamicin, 54.0\% with doxycycline, $59.4 \%$ with ciprofloxacin and $67.6 \%$ with cefaperazone /sulbactam. Resistance with meropenem was $10.8 \%$. Among the urinary isolates a resistance of $30 \%$ and $80 \%$ was observed with nitrofurantoin and norfloxacin respectively. (Fig4) 
During the past decade, ESBL producing gram negative bacilli especially $E$. coli and Klebsiella pneumoniae have emerged globally as major causes of hospital and community acquired infections. In our study $248 / 412(60.2 \%)$ isolates were found to be ESBL producers. Out of these, 84/248 $(33.9 \%)$ were from surgical ward, 49 (19.7\%) from outpatient department, 38/248 $(15.3 \%)$ from medicine ward, 36/248 $(14.5 \%)$ from pediatric ward, 35/248 (14.1\%) from ICU patients and 6/248 (2.4\%) from gynaecological ward. The prevalence of ESBLs among E. coli and Klebsiella pneumoniae was $61.9 \%$ and $56.5 \%$ respectively. The prevalence of ESBL among clinical isolates varies greatly worldwide and in geographical areas and is rapidly changing over time. Across India reports of ESBL detection among clinical isolates of $E$. coli range between $23.4 \%$ and $63.8 \%$ and those among Klebsiella pneumoniae ranges between $17.0 \%$ and $76.2 \%$.

Table.1 Distribution of ESBL producing E. coli and Klebsiella pneumoniae in different Wards, ICU and OPD

\begin{tabular}{|l|l|l|l|l|}
\hline \multirow{2}{*}{$\begin{array}{l}\text { Name of } \\
\text { Department }\end{array}$} & \multicolumn{3}{|l|}{ E. coli isolates } & \multicolumn{2}{l|}{ Klebsiella pneumoniae isolates } \\
\cline { 2 - 5 } & Total & $\begin{array}{l}\text { ESBL producers } \\
\text { No. }(\%)\end{array}$ & Total & $\begin{array}{l}\text { ESBL producers } \\
\text { No. }(\%)\end{array}$ \\
\hline OPD & 70 & $40(57.1)$ & 19 & $9(47.4)$ \\
\hline ICU & 18 & $14(77.8)$ & 32 & $21(65.6)$ \\
\hline $\begin{array}{l}\text { Surgical } \\
\text { ward }\end{array}$ & 100 & $69(69.0)$ & 32 & $15(46.9)$ \\
\hline $\begin{array}{l}\text { Medical } \\
\text { ward }\end{array}$ & 60 & $30(50.0)$ & 16 & $8(50.0)$ \\
\hline $\begin{array}{l}\text { Paediatric } \\
\text { ward }\end{array}$ & 25 & $16(64.0)$ & 29 & $20(69.0)$ \\
\hline $\begin{array}{l}\text { Obstretic } \\
\text { Gynae } \\
\text { ward }\end{array}$ & 8 & $5(62.5)$ & 3 & $1(33.3)$ \\
\hline Total & 281 & $174(61.9)$ & 131 & $74(56.5)$ \\
\hline
\end{tabular}

Table.2 Age and Gender distribution of ESBL producing E. coli and Klebsiella pneumoniae

\begin{tabular}{|l|l|l|l|l|}
\hline Age (years) & $\begin{array}{l}\text { Total No. of } \\
\text { isolates }\end{array}$ & \multicolumn{3}{|c|}{ ESBL producers } \\
\cline { 3 - 5 } & & $\begin{array}{l}\text { E. coli } \\
\text { No. }(\%)\end{array}$ & $\begin{array}{l}\text { Klebsiella } \\
\text { pneumoniae } \\
\text { No. }(\%)\end{array}$ & $\begin{array}{l}\text { Total } \\
\text { No. }(\%)\end{array}$ \\
\hline $0-14$ & 54 & $16(29.6)$ & $20(37.0)$ & $36(66.7)$ \\
\hline $15-44$ & 195 & $88(45.1)$ & $34(17.4)$ & $122(62.6)$ \\
\hline$>45$ & 163 & $70(42.9)$ & $20(12.3)$ & $90(55.2)$ \\
\hline Total & 412 & $174(42.2)$ & $74(18.0)$ & $248(60.2)$ \\
\hline Gender & \multicolumn{5}{|l|}{} \\
\hline Male & 279 & $123(44.08)$ & $40(14.3)$ & $163(58.4)$ \\
\hline Female & 133 & $51(38.34)$ & $34(25.6)$ & $85(63.9)$ \\
\hline Total & 412 & $174(42.23)$ & $74(18.0)$ & $248(60.2)$ \\
\hline
\end{tabular}


Table.3 Distribution of ESBL E. coli and Klebsiella pneumoniae from patients infected at various sites

\begin{tabular}{|l|l|l|l|l|l|l|l|}
\hline \multirow{2}{*}{ Site of infection } & \multicolumn{4}{|l|}{ E. coli } & \multicolumn{2}{l|}{ Klebsiella pneumoniae } & Total \\
\cline { 2 - 8 } & $\begin{array}{l}\text { In } \\
\text { patient } \%\end{array}$ & $\begin{array}{l}\text { Out } \\
\text { patient } \%\end{array}$ & $\begin{array}{l}\text { Total } \\
\%\end{array}$ & $\begin{array}{l}\text { In patient } \\
\%\end{array}$ & $\begin{array}{l}\text { Out } \\
\text { patient } \%\end{array}$ & $\begin{array}{l}\text { Total } \\
\%\end{array}$ & $\begin{array}{l}\text { ESBL } \\
\%\end{array}$ \\
\hline Respiratory tract* & 75.0 & 66.7 & 72.7 & 33.3 & 0 & 31.2 & 48.1 \\
\hline Urinary tract $\dagger$ & 65.4 & 62.1 & 64.5 & 42.8 & 70.0 & 58.8 & 64.1 \\
\hline Bacteremia & 90.9 & 0 & 83.3 & 80.0 & 0 & 78.0 & 79.2 \\
\hline Skin and soft tissue & 38.8 & 16.7 & 33.3 & 57.1 & 40.0 & 54.5 & 45.6 \\
\hline Others§ & 41.7 & 50.0 & 42.8 & 40.9 & 0 & 37.5 & 39.5 \\
\hline Total & 63.5 & 57.2 & 61.9 & 58.0 & 47.4 & 56.5 & 60.2 \\
\hline
\end{tabular}

*infections included pneumonia, tracheobronchitis and upper respiratory tract infection $\dagger$ infections included cystitis, prostatitis and pyelonephritis †infections included cellulitis,abcess and chronic ulcer infection $\S$ Infections included meningitis, encephalitis, pelvic inflammatory disease and intraabdominal infections

Fig.1 Phenotypic Confirmatory Disc Diffusion Test. ESBL production confirmed by an increase in zone of $\geq 5 \mathrm{~mm}$ for ceftazidime(CAZ) and ceftazidime/clavulanic acid(CAC) and cefotaxime(CTX) and cefotaxime/clavulanic acid(CEC)

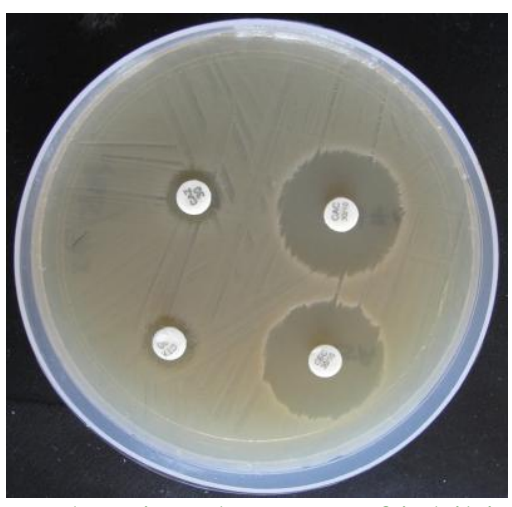

Fig.2 Double disc synergy test showing the zone of inhibition of ceftriaxone(CTR) and cefotaxime(CTX) enhancing towards the amoxycillin/clavulanic acid(AMC) disc containing disc confirming an ESBL producer

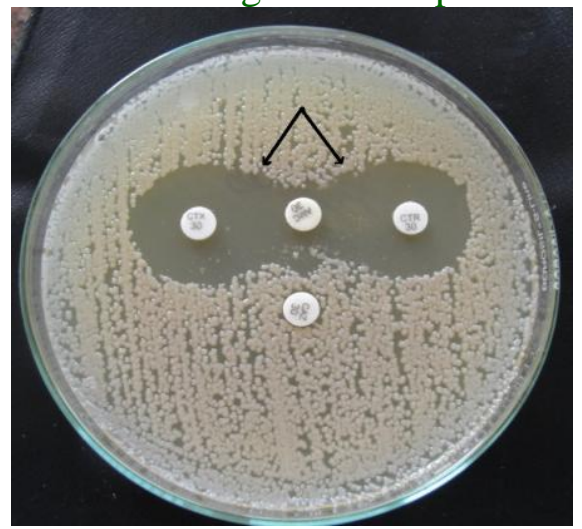


Fig.3 Antibiotic resistance pattern of ESBL positive isolates of E. coli among in-patients and out-patients

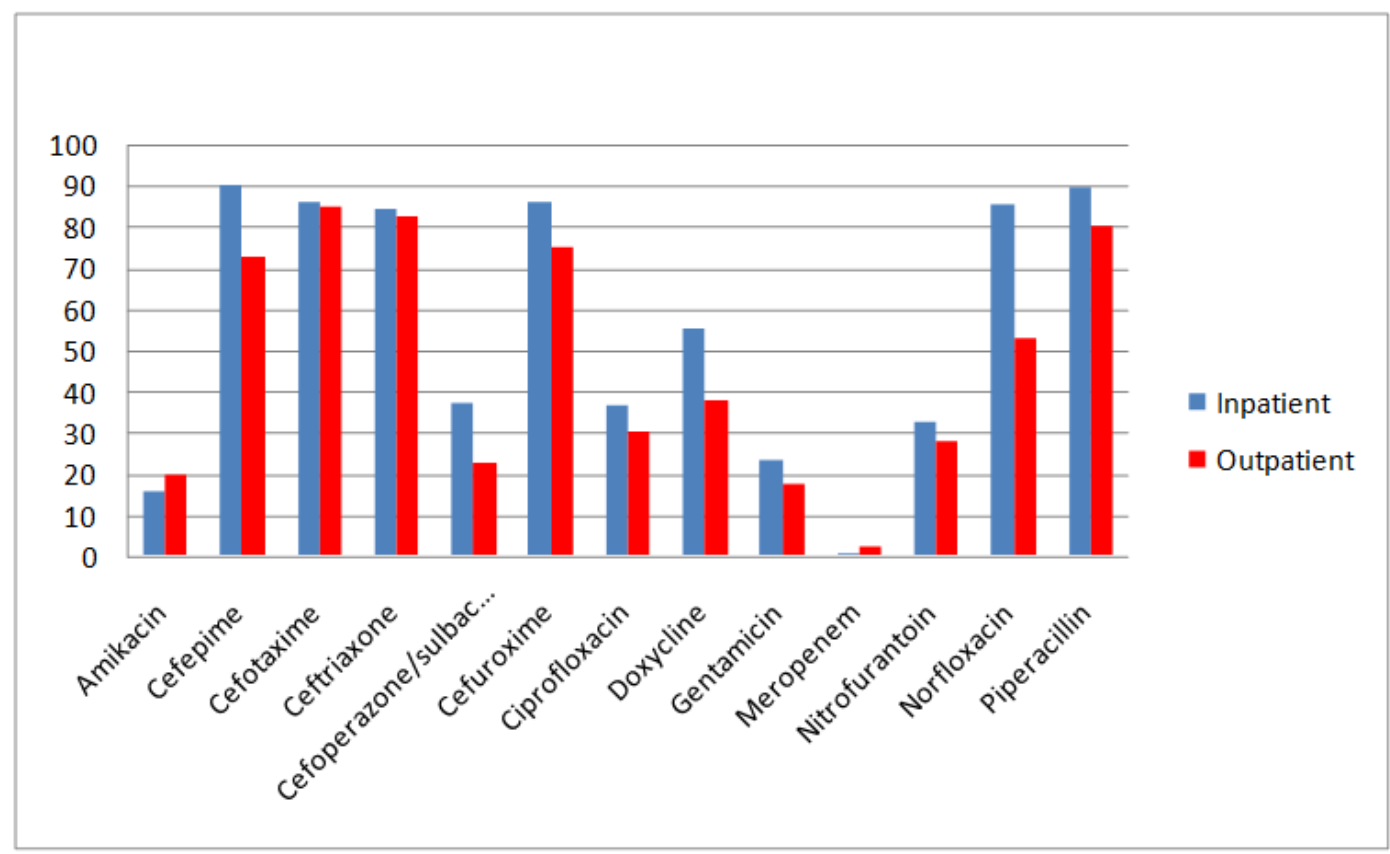

Fig.4 Antibiotic resistance pattern of ESBL positive isolates of Klebsiella pneumoniae among in-patients and out-patients

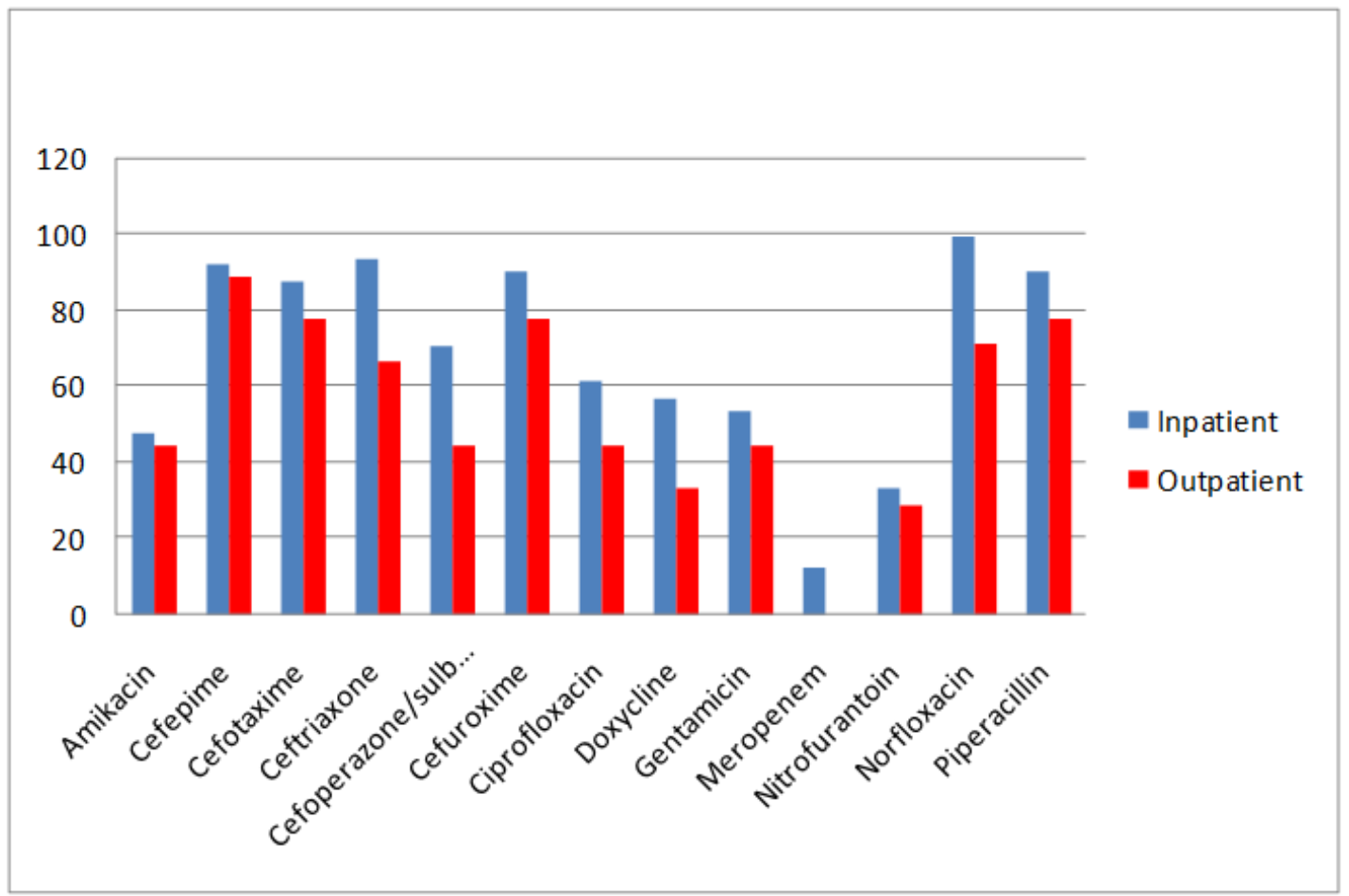


In the present study, the occurrence of ESBL was higher in hospitalized patients $199 / 323(61.6 \%)$ as compared to outpatients $(55.0 \%)$ though not statistically significant $(\mathrm{p}=0.26)$ and is in agreement with findings of other investigators.( Abhilash et al., 2010; Kader et al., 2005) Among the ESBL E. coli isolates highest occurrence was seen in ICU patients $14 / 18(77.8 \%)$ and surgical ward patients 69/100(69.0\%). Our study is in accordance with other studies. (Ashrafian $e t$ al., 2013; Babypadmini et al., 2004) The reason for this could be that patients in ICU tend to be more debilitated, are likely to have a higher use of invasive devices, more likely to need ventilatory assistance and have greater exposure to antimicrobial agents. The risk factors in surgery patients include catheterization and use of broad spectrum antibiotics preoperatively. Secondly in patients who have undergone surgery the duration of hospital stay may be a predisposing factor for colonization of ESBL producing organisms. Among the ESBL Klebsiella pneumoniae isolates highest occurrence was seen in pediatric patients 20/29(69.0\%) and ICU patients $21 / 32(65.6 \%)$.Among the pediatric patients majority were neonates18/20(90.0\%).The risk factors in these patients include low birth weight, prior exposure to antibiotics, use of invasive devices, underlying illness and length of hospital stay. (Vijayakanthi $e t$ al., 2013) The risk factors for acquiring ESBL Klebsiella pneumoniae in ICU patients was essentially the same as for ESBL E. coli.

In the present study highest percentage of ESBL E. coli was reported from patients of primary bacteremia followed by samples from respiratory tract infection and urinary tract infection. However, in other studies highest occurrence was seen in patients with urinary tract infection. (Grover et al., 2013)
Among ESBL Klebsiella pneumoniae highest percentage was reported in patients of primary bacteremia followed by urinary tract infection and skin and soft tissue infections. Sarojamma et al., (2011) have also reported a high percentage in blood samples. Similarly Gupta et al., (2007) have also reported $69.2 \%$ in their blood isolates. (Gupta et al., 2007) The occurrence of ESBL in bacteremic patients is of concern as many of these patients are hospitalized and cephalosporins are given empirically as first line agents. Delayed recognition and inappropriate treatment of severe infections caused by ESBL producers with cephalosporin has been associated with increased mortality.

In the present study a high $(\geq 80 \%)$ degree of resistance was seen with $3 \mathrm{GCs}$, cefepime, cefuroxime, piperacillin and norfloxacin in ESBL E. coli as well as Klebsiella pneumoniae strains. Our study is in agreement with several previous reports from various regions of the country. (Najam et al., 2015) Plasmids bearing genesencoding ESBLs frequently also carry genes encoding resistance to other antimicrobial agents, such as aminoglycosides, trimethoprim, sulphonamides, tetracyclines and chloramphenicol thus limiting therapeutic options. A moderate resistance of $40.8 \%$ and $51.1 \%$ was seen with gentamicin and ciprofloxacin in ESBL $E$. coli while it was $52.7 \%$ and $59.4 \%$ respectively in ESBL Klebsiella pneumoniae isolates. Other studies have reported variable rates of resistance to these antibiotics. (Sarojamma et al., 2011; Singh et al., 2011) Amikacin was more active against ESBL $E$. coli than Klebsiella pneumoniae isolates. Only $17.0 \%$ resistance was seen with nitrofurantoin in urinary isolates of ESBL $E$. coli. This is in accordance with various other studies. Carbapenems are often the last line of effective treatment available for critically 
ill patients. In the present study meropenem resistance was high (10.8\%) among ESBL Klebsiella pneumoniae isolates that reveals a serious threat to clinicians compromising treatment choices. Carbapenem resistance is usually multifactorial. Resistance to carbapenems occurs through bacterial production of $\beta$-lactamase enzymes that hydrolyze the antibacterial agent or through porin changes in the bacterial cell wall that reduce the permeability of the drug into the organism. In addition, upregulation of efflux pumps result into reduced susceptibility of organisms toward meropenem. (RodríguezMartínez et al., 2009)The options for treating carbapenem resistant organisms are limited and warrants close monitoring of resistance by microbiology laboratories.

The limitation of our study is that molecular characterization of isolates could not be done due to limited resources.

In conclusion, the prevalence of ESBL in the present study was high. Even though the occurrence of ESBL was higher in inpatients, it was only marginally less in outpatients. This should alert the clinicians as ESBLs are spreading fast in the community and may pose therapeutic challenges. This emphasizes the need for active surveillance for microorganisms producing ESBL $\beta$-lactamases which can help in providing appropriate antimicrobial therapy. Good infection control practices and antibiotic management interventions are instrumental in preventing the emergence of outbreaks due to ESBL producing isolates, especially in high risk areas such as the medical ICU, pediatric wards and surgical wards. Educational programs for medical staff to increase awareness of ESBLs should also be developed. Furthermore, increased empirical use of carbapenems in response to outbreaks of ESBL Klebsiella pneumoniae infections has been accompanied by the rapid emergence of carbapenem resistance in other nosocomial pathogens. Therefore, therapeutic options other than carbapenems should be made available. Tigecycline, colistin and polymyxin should be reserved for patients resistant to all of the other antibiotics, including the carbapenems. Further studies are needed to determine the epidemiology of ESBL related infections and the associated clinical burden locally.

\section{References}

Abhilash, K.P., Veeraraghavan, B., Abraham, O.C. 2010.Epidemiology and outcome of bacteremia caused by extended spectrum beta-lactamase (ESBL)-producing Escherichia coli and Klebsiella spp. in a tertiary care teaching hospital in south India. J. Assoc. Physicians India, 58(Suppl):13-7.

Ashrafian, F., Askari, E., Kalamatizade, E., Shahroodi, M.J.G., Naderi-Nasab, M. 2013.The Frequency of Extended Spectrum Beta Lactamase (ESBL) in Escherichia coli and Klebsiella pneumoniae, A Report from Mashhad. Iran. J. Med. Bacteriol., 2(1-2):12-9.

Babypadmini, S., Appalaraju, B. 2004. Extended spectrum- lactamases in urinary isolates of Escherichia coli and Klebsiella pneumoniae prevalence and susceptibility pattern in a tertiary care hospital. Indian J. Medical Microbiol., 22(3): 172-4.

Bradford, P.A. 2001. Extended-spectrumlactamases in the $21^{\text {st }}$ century: Characterization, epidemiology, and detection of this important resistance threat. Clin. Microbiol. Rev., 14(4): 933-51.

Chauhan, S., Mahawal, B.S., Ramola, D.C. 2015. Extended spectrum $\beta$ lactamases in urinary isolates of 
Escherichia coli - prevalence and susceptibility pattern at a tertiary care hospital. Int. J. Res. Med. Sci., 3(7): 1622-6.

Clinical Laboratory Standards Institute. 2015. Performance Standard for Antimicrobial Susceptibility Testing; twenty fifth Informational Supplement ed. CLSI Document M100-S25. Wayne, PA: CLSI.

Gupta, V., Singhla, N., Chander, J. 2007. Detection of ESBLs using third \& fourth generation cephalosporins in double disc synergy test Indian $J$. Med. Res., 126(5): 486-7.

Grover, N., Sahni, A.K., Bhattacharya, S. 2013. Therapeutic challenges of ESBLS and AmpC beta-lactamase producers in a tertiary care center. Armed Forces Med. J. India, 69: 410.

Jarlier, V., Nicolas, M., Fournier, G., Philippon, A. 1988.Extended broadspectrum beta-lactamases conferring transferable resistance to newer $\beta$ lactam agents in Enterobacteriaceae; hospital prevalence and susceptibility patterns. Rev. Infect. Dis., 10(4): 867-78.

Kader, A.A., Angamuthu, K. 2005. Extended-spectrum $\beta$-lactamases in urinary isolates of Escherichia coli, Klebsiella pneumoniae and other gram-negative bacteria in a hospital in Eastern Province, Saudi Arabia. Saudi Med. J., 26(6):956-9.

Kenneth, T. 2008. Bacterial Resistance to Antibiotics. Todar's Online Textbook of Bacteriology.

Khanfar, H.S., Bindayna, K.M., Senok, A.C., Botta, G.A. 2009. Extended spectrum beta-lactamases (ESBL) in Escherichia coli and Klebsiella pneumoniae: trends in the hospital and community settings.J. Infect. Dev. Ctries., 3(4): 295-9.
Laghawe, A., Jaitly, N., Thombare, V. 2012.The simultaneous detection of the ESBL and the AmpC blactamases in gram negative bacilli. JCDR. 6:660-63.

Metri, B.C., Jyothi, P., Peerapur, B.V. 2011. The prevalence of ESBL among Enterobacteraceae in a tertiary care hospital in North Karnataka, India. $J$. Clin. Diagn. Res., 5(3): 470-5.

Najam, M., Koppad, M., Halesh, S.H., Siddesh, K.C. 2015. Detection of Carbapenem Resistance in Extended Spectrum Beta Lactamase Producing Eshcherichia coli Isolates in a Tertiary Care Hospital. IJMR, 2(3):138-41.

Niranjan, V., Malini, A. 2014. Antimicrobial resistance pattern in Escherichia coli causing urinary tract infection among inpatients. Indian J. Med. Res., 139(6): 945-8.

Paterson, D.L. 2000. Recommendation for treatment of severe infections caused by Enterobacteriaceae producing extended-spectrum $\quad \beta$-lactamases (ESBLs) Clin. Microbiol. Infect., 6(9): 460-3.

Paterson, D.L., Bonomo, R.A. 2005. Extended-spectrum $\beta$-lactamases: A clinical update. Clin. Microbiol. Rev., 18(4):657-86.

Paterson, D.L. 2006. Resistance in gramnegative bacteria: Enterobacteriaceae. Am. J. Infect. Control. 34(Suppl1): S20-8.

Rodríguez-Martínez, J.M., Poirel, L., Nordmann, P. 2009. Molecular epidemiology and mechanisms of carbapenem resistance in Pseudomonas aeruginosa. Antimicrob. Agents Chemother., 53(11):4783-8

Sahni, R.D., Balaji, V., Varghese, R., John, J., Tansarli, G.S., Falagas, M.E. 2013. Evaluation of fosfomycin 
activity against uropathogens in a fosfomycin-naive population in South India: a prospective study. Future Microbiol., 8(5): 675-80.

Sarojamma, V., Ramakrishna, V. 2011. Prevalence of ESBL-

Producing Klebsiella

pneumoniae Isolates in Tertiary Care Hospital. ISRN Microbiol., 318348. doi:10.5402/2011/318348.

Shaikh, S., Fatima, J., Shakil, S., Rizv, S.M.D., Kamal, M.A. 2015. Risk factors for acquisition of extended spectrum bata lactamase producing Escherichia coli and Klebsiella pneumoniae in North-Indian hospitals. Saudi J. Biol. Sci., 22(1): 37-41.

Singh, R.E., Veena, M., Raghukumar, K.G., Vishwanath, G., Rao, P.N., Murlimanju, B.V. 2011. ESBL production: Resistance pattern in Escherichia coli and Klebsiella pneumoniae, a study by DDST method. Int. J. Appl. Biol. Pharm. Technol., 2(4): 415-22.

Umadevi, S., Kandhakumari, G., Joseph, N.M., Kumar, S., Easow, J.M., Stephen, S., et al., 2011. Prevalence and antimicrobial susceptibility pattern of ESBL producing Gram Negative Bacilli J. Clin. Diagn. Res., 5(2): 236-9.

Vijayakanthi, N., Bahl, D., Kaur, N., Maria, A., Dubey N.K.1. 2013.Frequency and characteristics of infections caused by extended-spectrum betalactamase-producing organisms in neonates: a prospective cohort study. Biomed. Res. Int., 756209.

Wani, K.A., Thakur, M.A., Fayaz, A., Fomdia, B., Gulnaz, B., Maroof, P. 2009. Extended spectrum - $\beta$ lactamase mediated resistance in Escherichia coli in a tertiary care hospital. Int. J. Health Sci., (Qassim), 3(2): 155-63.

\section{How to cite this article:}

Saroj Hooja, Nita Pal, Rajendra Karadiya, Rajni Sharma, Ramesh Mishra and Rakesh Maheshwari. 2016. Prevalence and Antimicrobial Susceptibility of Extended Spectrum $\beta$ Lactamases (ESBL) Producing Escherichia coli and Klebsiella pneumoniae Isolates in a Tertiary Care Hospital in North-West India. Int.J.Curr.Microbiol.App.Sci. 55): 430-439. doi: http://dx.doi.org/10.20546/ijcmas.2016.505.045 